ÚRSULA BRAVO

FACULTAD DE DISEÑO

UNIVERSIDAD DEL DESARROLLO

SANTIAGO, CHILE

UBRAVO@UDD.CL
Parte de este trabajo fue presentado

en el III Encuentro Iberoamericano en Políticas, Gestión e Industrias Culturales (Santiago de Chile, Mayo de 2105), y en el VII Congreso Latinoamericano de Enseñanza del Diseño (Buenos Aires, Julio de 2016).

Fecha de recepción: 30/03/2015 Fecha de aceptación: 12/04/2016 Cómo citar: Bravo, Ú. (2016). Diseño para el desarrollo. Vigencia de los principios de UIm frente a los desafíos del proyecto país. RChD: creación y pensamiento, 1(1), 79-93. DOI: 10.5354/0718-2430.2016.44314

\section{Diseño para el desarrollo. Vigencia de los principios de Ulm frente a los desafíos del proyecto país}

\author{
Design for Development. Effectiveness of UIm Principles Facing \\ the Challenges of the Country Project
}

Resumen. Desde hace algún tiempo podemos observar una transición desde iniciativas estatales orientadas a fomentar la difusión del diseño chileno, hacia iniciativas que fomentan su aplicación en diversos sectores de la vida social, con el propósito de generar beneficios sociales o económicos. Por ello cabe preguntarse cuál es el rol que el Estado chileno asigna al diseño en la sociedad que aspira construir y cuán alineados están los planes de estudios con estos requerimientos. A casi cincuenta años de su cierre, es necesario redescubrir la $\mathrm{HfG}-U \mathrm{~lm}$ para entender los principios con los que desarrolló un modelo educativo que se distanciaba de las escuelas de artes y oficios, para responder a la reconstrucción moral y material del país. Este trabajo busca analizar esta propuesta pedagógica como forma de entender el diseño y su inserción en el tejido social. La reflexión cobra vigencia porque si bien el Estado chileno ha ido incorporando al diseño en distintas iniciativas, todavía está en construcción una visión integrada de lo que el diseño es y puede llegar $a$-hacer por el país. Por otra parte, la pregunta acerca del propósito de la formación disciplinar dista de estar resuelta y generar consenso.

Palabras clave: currículum de diseño, diseño, escuela de Ulm, políticas públicas.

\begin{abstract}
Since some time it can be observed a transition from state initiatives led to promote the dissemination of Chilean design, to initiatives that encourage its application in diverse sectors of social life, aiming to generate social and economic benefits. Hence, the question comes about on what is the role the Chilean State allocates upon Design in the society that aspires to build and how the academic curricula are aligned with these requirements. About fifty years after its closure, it becomes necessary to rediscover the $\mathrm{HfG}-U \mathrm{~lm}$ to understand the principles employed to develop its educational model, distancing itself from arts and crafts schools and responding to the moral and material reconstruction of the country. This paper seeks to analyse this pedagogical proposal as a way to understand Design and its integration into the social frame. The reflection becomes effective because although, the Chilean State has incorporated Design into various initiatives; a unified vision of what design is, and can get to do for the country still is under construction. Moreover, the question about the purpose of disciplinary training is far from resolved and generate consensus.
\end{abstract} Keywords: design, design curriculum, HfG-Ulm, public policies.

Revista Chilena de Diseño,

RChD: creación y pensamiento

Universidad de Chile

2016, I(1)

http://rchd.uchile.cl 
1. Ver <http://www.cultura.gob.cl/

institucionales/comite-interministerial -aprueba-lineamientos-que-regiran-el-plan -nacional-de-economia-creativa/>

2. Hfc es la sigla para referirse a la Hochschule für Gestaltung o Escuela Superior de Proyectación de la ciudad de UIm. Para efecto de este artículo hablaremos indistintamente de HfG, Escuela de UIm o simplemente UIm.

\section{Introducción}

En los últimos años diversas iniciativas provenientes del Estado incluyen al diseño como factor de desarrollo económico, social y cultural. Desde la CORFO, el Fondo Capital Humano para la Innovación en Pymes fue creado como medida para la reactivación económica con el doble propósito de aumentar la competitividad de las pymes y fomentar la empleabilidad. El Laboratorio de Gobierno busca formalizar una instancia para probar, pilotear y escalar ideas innovadoras que agreguen valor a los servicios del Estado. El Consejo Nacional de la Cultura y las Artes (CNCA), por su parte, recibió la misión de liderar dos importantes iniciativas: la elaboración de un Plan Nacional de Economía Creativa que permita incrementar el aporte del sector al PIB $^{1}$ y la elaboración de una Política Nacional de Diseño. Tanto el Laboratorio de Gobierno como el Plan Nacional de Economía Creativa incluyen equipos interministeriales que se espera contribuyan a generar una mirada integradora y ampliar la cobertura de estas iniciativas hacia distintos sectores del Estado. Estas iniciativas estarían señalando el inicio de una nueva etapa de desarrollo del diseño chileno y podrían ser consideradas como resultado de un esfuerzo sistemático realizado por diferentes representantes del diseño nacional que, desde las asociaciones profesionales, la academia, el mundo empresarial y el Área de Diseño del CNCA, han trabajado arduamente para posicionar la disciplina.

Esta nueva etapa estaría dada por una transición desde iniciativas estatales orientadas a fomentar la creación, la difusión y el fortalecimiento disciplinar, hacia iniciativas que fomentan su aplicación sistemática en diversos sectores de la vida social, con el propósito de introducir mejoras que se traduzcan en beneficios sociales y/o económicos, confirmando la visión del diseño como factor de desarrollo, propuesta por la ıv Bienal de Diseño, en el marco del Bicentenario.

El vínculo entre diseño y desarrollo no es una novedad para quienes habitamos esta disciplina, la novedad está en que el Estado haya comenzado a realizar acciones que confirman esta visión. En este contexto, cabe preguntarse cuál es el rol que el Estado chileno asigna al diseño en la sociedad que aspira construir y cuán alineadas están las iniciativas provenientes de sus distintos organismos. Asimismo, desde la academia debemos preguntarnos si los planes de estudios están respondiendo a estos nuevos requerimientos. Frente a estas interrogantes, cabe recordar que a partir de 1958 la $\mathrm{HfG}_{-} \mathrm{Ulm}^{2}$ implementó un modelo educativo que se distanciaba de la tradición de las escuelas de artes y oficios, buscando articular la reconstrucción moral y material del país con la misión de constituir el diseño como una disciplina autónoma. Para ello racionalizó un método proyectual, incorporó materias teóricas provenientes de las ciencias humanas y exactas, y estableció una estrecha colaboración con la industria y con diversos actores del ámbito profesional y académico.

A casi cincuenta años de su cierre, es necesario preguntarse por la vigencia de esta mítica escuela: ¿constituye solo un interesante objeto de estudio para la historiografía del diseño o aún es posible rescatar de aquella experiencia algunos principios que orienten las estrategias de fortalecimiento 
disciplinar, el diseño de programas académicos y la incorporación progresiva del diseño a distintos ámbitos de la vida social?

Lejos de querer hacer una apología a Ulm, este trabajo busca analizar su propuesta pedagógica como forma de entender el diseño y su inserción en el tejido social, enfatizando el rol mediador que lo distancia de la expresión artística. La reflexión cobra vigencia porque si bien el Estado chileno ha ido incorporando al diseño en distintas iniciativas, todavía está en construcción una visión integrada de lo que el diseño es y puede llegar $a$-hacer por el país. Iniciativas como el foro sobre el nuevo perfil profesional de los diseñadores, organizado por Chile Diseño a fines de 2014 - que congregó a académicos y directivos de distintas universidades - pone de manifiesto que la pregunta acerca del propósito de la formación disciplinar dista de estar resuelta y generar consenso. Para desarrollar este trabajo se realizó una revisión bibliográfica que incluyó libros y artículos, así como informes y documentos de distintas organizaciones. La investigación bibliográfica fue complementada con entrevistas a Manuel Figueroa, coordinador del Área de Diseño del CNCA, y a los académicos Katherine Mollenhauer y Rodrigo Ramírez, que colaboraron en el diseño de la futura Política Nacional de Diseño.

\section{Ulm: una propuesta académica para la construcción social y el desarrollo disciplinar}

No es posible comprender el significado de UIm, sin considerar las circunstancias que motivaron su creación y el contexto sociopolítico y económico en el que se desarrolló. Este determinó una particular manera de entender la función del diseño en la sociedad, lo que se expresó a nivel de desarrollo disciplinar y de propuesta curricular. Es así como es posible identificar un modelo triádico en el que se articulan los siguientes componentes:

a. Una visión país o modelo social que se aspira alcanzar.

b. La definición de un perfil profesional y el diseño de un programa académico que permita entregar los conocimientos y desarrollar las habilidades y los valores necesarios para la formación de un profesional que pueda contribuir a alcanzar dicho modelo social.

c. Una determinada visión disciplinar que oriente el desarrollo de un corpus teórico, así como también iniciativas de asociatividad y de vinculación con el sector profesional, académico y productivo.

Este modelo no pierde vigencia, en la medida en que se adapta a cualquier contexto histórico, político y económico, toda vez que intenta responder a interrogantes fundamentales (ver tabla en página siguiente).

\section{Dimensión social. Diseño para la reconstrucción moral y material}

Al igual que la Bauhaus, Ulm se funda en un contexto de posguerra, donde la destrucción material imponía tareas impostergables y la derrota moral exigía la búsqueda de nuevas certezas. A diferencia de la Bauhaus, Ulm va tomando progresiva distancia del arte, cuestionando su validez desde una perspectiva moral y metodológica. Aicher lo expresa de la siguiente manera:

Habíamos regresado de la guerra, y en la academia nos encontrábamos con que debíamos trabajar en la estética por la estética. Aquello no podía seguir. Quienquiera que tenía ojos para 


$\begin{array}{lll} & & \\ & \text { ¿Cuál es el país que queremos } \\ & \text { Construir? }\end{array}$

contribuir a ello?

profesional

PROFESIÓN

Formación

¿Cuáles son los conocimientos que

profesional

debe adquirir?

Programa

académico

¿Cuáles son las habilidades que debe

desarrollar?

¿Cuál es la formación valórica que

le permitirá autorregularse?

¿Cuál el sustento teórico y

metodológico de la disciplina?

Desarrollo teórico

y metodológico de

la disciplina

Vinculación y

DISCIPLINA generación de

redes

Posicionamiento

frente a diferentes

estamentos de la

sociedad
¿Qué cuerpo de conocimientos

fundamentan, amplían y nutren

la práctica profesional?

¿Cuáles son las metodologías que otorgan validez a la disciplina?

¿Cómo sistematizar el conocimiento implícito en el ejercicio profesional, de manera de hacerlo replicable y enseñable?

¿Cuáles son los actores relevantes de la disciplina y cómo generar sinergias en beneficio de ésta? 
ver y oídos para oír, hubo de reconocer que el arte era una huida de las múltiples tareas que aguardaban, también a la cultura, tras el derrumbe del régimen nazi. (Aicher, 1997, p.81)

Desde su gestación, la reconstrucción material fue considerada un problema moral que incumbía directamente a la academia. En sus notas solicitando financiamiento al Alto Comisionado de Estados Unidos, Inge Scholl apela al sentido de responsabilidad de los jóvenes intelectuales alemanes respecto del futuro espiritual, económico y político del país, señalando el rol fundamental que debía tener la universidad para llevar cabo dicha tarea (Lindinger, 1991, p.18). Así, desde su origen, Ulm entendió el diseño como medio para impulsar el desarrollo; visión que habría resultado fundamental para que finalmente se optara por la creación de una escuela de diseño y no una de educación cívica (Quijano, 2003, p.11). Según el exalumno y profesor, Gui Bonsiepe, "el programa ulmiano estaba estrechamente ligado al proyecto del iluminismo y contenía un componente utópico, cuyo abandono habría implicado cinismo y pérdida de esperanza" (Bonsiepe, 1999, p.136).3 En un artículo publicado en 1955, Maldonado afirmaba:

La Escuela de Ulm quiere señalar el camino a seguir para lograr el más alto nivel de creatividad, pero a la vez, y no en menor medida, señalar cuál ha de ser la finalidad social de esta creatividad, es decir, indicar cuáles son las formas que merecen ser creadas y cuáles no. [...] En su programa, el acento ya no se pone más en lo moderno 'en general', sino en un tipo determinado de modernidad y de creatividad que destacan el contenido social tanto de una como de otra. [...] La Hfc hace suya la tesis según la cual el proyectista, aun trabajando para la industria, ha de continuar asumiendo sus responsabilidades frente a la sociedad. En ninguna circunstancia sus obligaciones para con la industria podrán anteponerse a sus obligaciones con la sociedad. (Maldonado, 1977, p.70)

Más allá del componente mesiánico que se evidencia en el texto de Maldonado, resulta necesario volver sobre estos cuestionamientos cuando ha quedado en evidencia la ineficiencia del mercado frente a demandas sociales como la salud o la educación. Tal como sostiene el economista francés Thomas Piketty:

El crecimiento moderno y la difusión de los conocimientos permitieron evitar el apocalipsis marxista, mas no modificaron las estructuras profundas del capital y de las desigualdades, o por lo menos no tanto como se imaginó en las décadas optimistas posteriores a la Segunda Guerra Mundial. [...] El capitalismo produce mecánicamente desigualdades insostenibles, arbitrarias, que cuestionan de modo radical los valores meritocráticos en los que se fundamentan nuestras sociedades democráticas. (Piketty, 2014, p.7)

De manera similar, en el contexto de las actuales políticas de fomento a la innovación y la productividad, el giro desde la noción de "competitividad" a la de "desarrollo" da cuenta de un nuevo énfasis que cuestiona la competitividad, entendida como un fin en sí mismo, recordándonos que el propósito de dichas políticas es alcanzar el desarrollo. ${ }^{4}$ Este giro puede ser muy positivo
3. A juicio del autor, esta dimensión de UIm

habría tenido un fuerte impacto entre los estudiantes latinoamericanos a fines de los años sesenta: “En Chile, Argentina, Brasil y México, los estudiantes no estaban solamente interesados en aprender las prácticas profesionales estándar, sino también en encontrar una respuesta a dos preguntas: ¿qué puede hacer un diseñador en la Periferia?, ¿cómo podría influir en la identidad cultural con su actividad?". Y agrega: "La respuesta a la segunda pregunta lleva directamente a la dimensión política que se puede sintetizar en la pregunta sobre el tipo de sociedad en la cual se quiere vivir" (Bonsiepe, 1999, p.124).

4. El antiguo "Consejo Nacional de Innovación para la Competitividad" hoy se Ilama "Consejo Nacional de Innovación para el Desarrollo” y la antigua "Gerencia de Competitividad" de CoRFo hoy es la "Gerencia de Desarrollo Competitivo". En el ámbito de las políticas culturales, esto se evidencia en el concepto de economía creativa, que ha reemplazado el de industria creativa. 
5. Se ha extendido la visión de la exagerada inclinación de Ulm por las ciencias y el método científico, sin embargo es necesario precisar que se dictaban alrededor de 210 horas de cursos teóricos -como Sociología, Psicología Social, Economía, Ciencias Políticas, Análisis de Operaciones Matemáticas, Teoría Estructural, Teoría de la Ciencia e Historia de la Cultura- en comparación con las 700 a 800 horas destinadas a trabajo práctico en cada especialidad (Mäntele, 2003, p.13). para el diseño, en la medida que genere oportunidades para su incorporación al ámbito público, aunque sin cuestionar su rol como factor que estimula la competitividad de los mercados mediante estrategias de diferenciación.

\section{Dimensión profesional. Perfil profesional y programa académico como catalizador}

Volviendo a Ulm, la reforma curricular liderada por Maldonado buscó sistematizar una metodología proyectual e incorporar materias teóricas, científicas y tecnológicas que permitieran a los estudiantes considerar una diversa gama de variables técnico productivas, conformar equipos de trabajo en conjunto con ingenieros y otros profesionales ligados a la industria y estar actualizados respecto de las posibilidades que brindaba el desarrollo tecnológico. De esta manera los problemas relativos a la forma de los objetos fueron abordados como una variable más dentro de muchas otras (Rinker, 2003, p.6).

En su búsqueda por objetivar el proceso proyectual, Ulm adhirió a la euforia científica de la década de 1950, clima que "hizo posible la formulación de una teoría del diseño caracterizada por la racionalidad". 5 Esto fue particularmente significativo, en la medida que "hasta este momento, no había habido ninguna sistematización del diseño" (Rinker, 2003, p.8). Según Bonsiepe, el programa de la institución pretendía "explorar el rico potencial de la ciencia, según el razonamiento asumido de que los conocimientos científicos podían enriquecer el proyecto y hacerlo más fundamentado" (Bonsiepe, 1999, p.139). Quijano afirma que UIm "contribuyó fundamentalmente en la consolidación de una metodología que ha tenido un efecto perdurable en la formación de diseñadores en todo el mundo" (Quijano, 2003, p.10). Bonsiepe, por su parte, sostiene que "Ulm objetivó la didáctica proyectual con la intención programática de construir un puente hacia las disciplinas científicas. Rompió con la larga tradición de los programas de enseñanza skill-oriented. La proyectación fue desmitificada y fue tratada como un dominio que se puede aprender $y$ enseñar con métodos" (Bonsiepe, 1999, p.137).

El programa académico de UIm influyó de manera decisiva en la definición de la identidad profesional del diseñador industrial y respondió a la necesidad de establecer nuevas distinciones con el propósito de alejarse de las categorías artísticas y expresivas con que el diseño había estado vinculado y que tenían su origen en las escuelas de artes y oficio. La propuesta de Maldonado tuvo un impacto directo en la caracterización presentada por el ICSID en 1960, donde se recogió la mayor parte de sus postulados. Esta definición no solo apelaba al desempeño técnico profesional, sino que incluía una dimensión ética al expresar que el objetivo de esta actividad "son productos industriales que sirvan a la sociedad en un sentido cultural y social". La escuela también contribuyó a ampliar la noción de diseño gráfico hacia la noción de comunicación visual, incorporando formas de comunicación no persuasiva, como sistemas de signos, planos para aparatos técnicos y visualización de contenidos científicos (Rinker, 2003, pp.5-8).

Lo interesante en Ulm es que los conocimientos científicos, técnicos y metodológicos orientados a objetivar el proceso de diseño, fueron complementados por cursos como Sociología, Psicología Social, Economía, Ciencias Políticas, Teoría de la Ciencia e Historia y Filosofía, que buscaban desarrollar un espíritu crítico y reflexivo, que permitiera tutelar desde una ética social, la práctica disciplinar. De esta manera, el programa de estudios no se centraba exclusivamente en el desarrollo de habilidades profesionales, sino 
que promovía una mirada crítica de la realidad, sustentada en el desarrollo intelectual. El diseñador debía ser, antes que todo, un ciudadano responsable, comprometido con el desarrollo económico, social y valórico de su entorno. Esta relación entre diseño y política es descrita por Bonsiepe en los siguientes términos:

Así como la dimensión estética es parte constitutiva del diseño, del mismo modo el componente político no puede ser desplazado de su campo de acción [...]. No se trata de atribuir al diseño un matiz político. Pero el diseño es inevitablemente político, porque comprende un componente de esperanza: el sueño aunque vago de una sociedad más digna de vivirse. Esta es una de las tesis irrenunciables de HfG-Ulm. (Bonsiepe, 1999, pp.139-140)

\section{Dimensión disciplinar. Desarrollo disciplinar y fomento del diseño}

La tercera dimensión que conforma este modelo, es la visión disciplinar y responde al propósito de considerar al diseño como disciplina autónoma. Aunque pueda parecer una obviedad que una escuela de diseño contenga a su vez la propuesta de una determinada visión disciplinar, en nuestro país la realidad de la oferta académica ${ }^{6}$ no permite discriminar con facilidad entre aquellos proyectos educativos que aportan al desarrollo disciplinar y los que solo se centran en la formación.?

Para ello Ulm contribuyó a delimitar el ámbito de acción del diseño, a desarrollar un cuerpo de conocimientos que permitiera fundamentarlo teóricamente y a sistematizar y modelar el conocimiento implícito en la práctica mediante metodologías que permitieran hacer del diseño una actividad objetivable, replicable y susceptible de ser enseñada.

El Departamento de Información, que corresponde a lo que hoy sería un Departamento de Teoría del Diseño, cumplió con el doble propósito de formar a diseñadores para la visualización de contenido científico -lo que hoy llamaríamos diseño de información-y de promover la reflexión teórica. En este programa se dictaba Historia, Organización y Teoría de los Media, Teoría de la Información, Lingüística, Historia de la Literatura Moderna, Semiótica, Sociología, además de Tipografía, Fotografía, Cine y Sonido (Lindinger, 1991, pp.208-283).

El aporte de Ulm al desarrollo de un cuerpo teórico puede ser constatado revisando la bibliografía de los actuales programas de diseño, donde abundan autores como Aicher, Albers, Archer, Bonsiepe, Dorfles, Moles, MüIler-Brockman, Maldonado y Rittel, que estuvieron directa o indirectamente vinculados a la escuela.

La creación de la revista Ulm constituyó una plataforma de difusión de sus postulados. En la introducción del primer número Maldonado insiste en el imperativo moral del diseño: lo reconoce como promotor del progreso científico y tecnológico, alerta sobre el rol instrumental al que puede ser relegado en una economía centrada en el mercado y le asigna al arte una función instrumental en el proceso proyectual (Bonsiepe, 2003, p.15).

Por otro lado, Ulm realizó distintas formas de vinculación: a nivel intradisciplinario, interdisciplinario y sectorial, con el propósito de posicionar al diseño en un nivel que le permitiera dialogar con soltura en contextos académicos, empresariales y estatales y dar a conocer su potencial transformador. El nivel intradisciplinario promueve redes de colaboración entre distintos actores
6. La oferta excesiva de programas académicos que imparten diseño, la diversidad de especialidades, los distintos tipos de certificados que se entregan -título técnico, título profesional y grado académico- y los distintos tipos de establecimientos que imparten diseño - centros de formación técnica, institutos profesionales y universidades-, generan un entorno complejo y ambiguo que no favorece a la disciplina. A esto se suma un sistema de acreditación de programas de pregrado que no define estándares de calidad diferenciados y un mercado de la educación superior que ha tendido a centrarse en el desarrollo de habilidades profesionales que permitan una rápida inserción en el ámbito productivo, desatendiendo en muchos casos la reflexión crítica y el desarrollo intelectual de los estudiantes.

7. Cabe recordar que la profesión es una actividad laboral especializada que se inserta en un determinado ámbito productivo formando parte de un tejido socioeconómico, mientras que la disciplina es un campo de estudio que incluye tanto la historia de dicha disciplina, como su cuerpo teórico y metodológico. 
del ámbito del diseño: profesionales, académicos, agrupaciones locales e internacionales. El nivel interdisciplinario busca vincularse con otros ámbitos disciplinares que contribuyan a desarrollar un cuerpo técnico, teórico y metodológico para el diseño. Por último, el nivel sectorial promueve la colaboración con otros estamentos de la sociedad como el sector público, el sector productivo y el empresarial.

Dan cuenta de las variadas formas de vinculación de Ulm: la participación en congresos y ferias, la organización de exhibiciones y la incorporación en la planta docente de figuras destacadas en sus campos disciplinares, como Bruce Archer, Abraham Moles, Max Bense, Horst Rittel y Josef Albers. Así como las visitas de exponentes del mundo del diseño y de la cultura, como las realizada por el diseñador estadounidense Charles Eames, el teórico de arte Gillo Dorfles y el filósofo alemán Martin Heidegger (Lindinger, 1991).

\section{Vigencia del modelo sociedad-profesión-disciplina en el contexto nacional} Desde hace un tiempo las instituciones públicas y privadas de nuestro país, están sufriendo una crisis de credibilidad que ha ido escalando al punto de que hoy es frecuente escuchar conceptos como crisis institucional, cambio de paradigma o nuevo pacto social: términos que aluden a una ciudadanía descontenta que cuestiona un modelo social que hasta ahora parecía ser más bien exitoso. La relaciones entre lo público y lo privado se tensionan y quedan en evidencia problemas de falta de probidad, uso de información privilegiada, tráfico de influencias y colusión, entre otras anomalías. Situaciones que cuestionan la independencia de los poderes del Estado o derechamente burlan la ley. Lo legal y lo ético no son equivalentes y los ciudadanos se sienten responsables de denunciar hechos que, pudiendo ser legales, no son éticos porque vulneran lo que se ha llamado "el espíritu de la ley". En un plano económico y social, pese al incremento de indicadores macroeconómicos como el PIB y el ingreso per cápita, los índices de desigualdad, el envejecimiento poblacional, la descentralización, el desarrollo de capital humano avanzado, la concentración de la canasta exportadora y la dependencia de la extracción de recursos naturales, son asignaturas pendientes. En este contexto, queda en evidencia una serie de demandas éticas, sociales y económicas a las que el país debe hacer frente. Guardando las proporciones respecto de lo que fue la reconstrucción alemana en la segunda posguerra, es válido volver sobre algunas de las preguntas fundamentales que se extraen del modelo sociedad-profesión-disciplina: ¿cuál es el tipo de sociedad en la que queremos vivir y de qué manera un diseñador puede contribuir a ello? En el ámbito público, estas preguntas derivan en nuevas interrogantes, como: ¿cuál es la visión que el Estado tiene del diseño?, ¿cuál es el rol que le asigna en el proyecto país que busca construir y cuáles son las acciones que emprende para lograrlo?

\section{Políticas culturales y diseño: ¿amenaza u oportunidad?}

En la última década, el organismo estatal que más ha contribuido a la promoción del diseño en Chile sin duda ha sido el CNCA a través del Área de Diseño, creada el año 2011. En estos años dicha área ha tenido un rol integrador y participativo, reuniendo a un sector que tradicionalmente ha estado poco vinculado. La elaboración de una Política Nacional de Diseño que tras sucesivas postergaciones espera ser presentada a mediados de 2017 , ha sido liderada por esta área y se sumará a las otras políticas sectoriales del CNCA. 
El equipo, a cargo de Manuel Figueroa, es accesible, tiene capacidad de convocatoria, está abierto a apoyar iniciativas que provengan de diversos actores del mundo académico y profesional. Su confirmación en el cargo después del último cambio de gobierno permitió dar continuidad a su gestión. ${ }^{8}$ Por estar inserto en una institucionalidad de la cultura, el Área de Diseño, necesariamente se rige por la política cultural vigente (2011-2016), y aunque se trata de un marco referencial suficientemente amplio como para acoger sectores creativos tan distintos como artes visuales, danza, artesanía y arquitectura, cabe preguntarse en qué medida los principios y objetivos de dicha política pueden contribuir al fortalecimiento e incorporación sistemática del diseño en los distintos ámbitos de la vida nacional. No es posible -y sería absurdo- desconocer el vínculo fundacional que el diseño tiene con el arte, sin embargo la relación entre ambos no ha estado exenta de controversias y, pese a la gran cantidad de literatura que lo aborda, es un tema que dista de estar resuelto o generar consensos. Es por esto que la institucionalidad del diseño ligada al ámbito artístico y cultural, merece ser analizada con detención, ya que de alguna manera expresa la visión que el Estado tiene de la disciplina.

La tendencia mundial desde la década de los noventa ha sido influida por el paradigma de la industria cultural o industria creativa o economía creativa. Aunque a veces puedan usarse indistintamente, estos términos no son sinónimos: ${ }^{9}$ sus diferencias generan controversias porque reflejan perspectivas y énfasis ideológicos distintos respecto del rol de la cultura. Son conceptos que han permeado desde la discusión sobre las políticas culturales, hasta la manera en que los mismos actores e instituciones culturales se refieren a sí mismos (PNUD y UNESCO, 2013, p.19). En términos generales, esta visión promueve un rol económico de la cultura y de la creatividad, afirmando que incentiva el emprendimiento, favorece la empleabilidad, incrementa las exportaciones y estimula la aparición de nuevas tecnologías. En síntesis, porque genera beneficios económicos.

En este contexto, no debe llamar la atención que revista Capital haya titulado su edición de enero de 2015 Creatividad: la deuda de Chile. En su presentación, el director de la publicación sostiene:

En un creciente número de economías desarrolladas, el encuentro entre creatividad, humanidades y la empresa es algo cada vez más común. Algo que no se expresa en la aparición y consolidación de emprendimientos creativos, sino que en la introducción de una cultura de innovación interna que está produciendo un círculo virtuoso. (Sapag, 2015, p.12)

Las políticas culturales en Chile no han escapado a esta influencia. En la primera Política Cultural del CNCA, ${ }^{10}$ José Weinstein afirmaba: "es necesario que la cultura sea aceptada ya no solo como generadora de valores estéticos y de sentido, sino también en tanto industria, generadora de riqueza y empleo e impulsora de nuevos horizontes para la economía del país"; reconociendo sus particularidades respecto de otros sectores económicos y su potencial de crecimiento (CNCA, 2005, p.3).

Por su parte, la actual Política Cultural 2011-201611 aspira a fortalecer "los mecanismos de participación social en su vida cultural [...], incentivando los procesos de participación, consumo y apropiación de bienes y servicios culturales, con un fuerte énfasis en la creación de hábitos de consumo en su
8. Otros organismos del Estado que fomentan el diseño son la Dirección de Asuntos Culturales (DIRAC) del Ministerio de Relaciones Exteriores de la República de Chile, ProChile, Sercotec y CORFO (CNCA, 2014, p.300).

9. El concepto "industrias culturales" es introducido en las décadas de 1930 y 1940, por la Escuela de Frankfurt, para caracterizar la degradación cultural generada por los medios de comunicación masiva en las sociedades capitalistas. En esta visión, cultura y economía tienen una relación hostil, en tanto la primera siempre se ve amenazada por la segunda. A partir de los años ochenta, el término empezó a ser utilizado como una calificación positiva, para referirse a formas de producción y consumo cultural que tenían en el centro un elemento expresivo o simbólico. Actualmente abarca campos tan diversos como la música, la escritura, la moda, el diseño y las industrias de los medios de comunicación. No tiene como referente obligado la producción industrial y considera la producción cultural de tipo artesanal. El concepto "industrias creativas" es más amplio, ya que, además de los bienes y servicios tradicionales de las industrias culturales, se abre a la innovación, incluyendo la investigación y desarrollo de software. La Unesco reconoce que "el uso de los términos 'industrias culturales y creativas' puede variar de forma significativa de un contexto a otro" y que están sujetos a una constante evolución. Finalmente, la noción de "economía creativa" es aún más amplia, porque "no solo abarca bienes y servicios culturales, sino también juguetes y juegos, así como todo el ámbito de "investigación y desarrollo" (I+D)". Si bien reconoce las actividades y los procesos culturales como el núcleo de una nueva economía, también "se ocupa de manifestaciones creativas en ámbitos que no serían contemplados como "culturales" (PNUD y UNESCO, 2013, pp.20-21).

10. El CNCA se crea en el gobierno del Presidente Lagos y su Política Cultural 2005-2010 se presenta en 2005, siendo ministro presidente del Consejo, José Weinstein.

11. Esta segunda política fue publicada en 2011 , durante el gobierno del Presidente Piñera, siendo ministro presidente del Consejo, Luciano Cruz-Coke. 
12. Publicación en formato digital del Departamento de Estudios del CNCA, que comenzó a circular en 2011. En su edición número 25 reúne una selección de artículos publicados a lo largo de los tres años de la revista. sociedad y en la participación de la vida cultural cotidiana" (CNCA, 2011, p.51). Dentro de sus objetivos se propone visibilizar y fomentar las industrias culturales como motor de desarrollo y contribuir a instalar los bienes y servicios artísticos y culturales en el escenario internacional (CNCA, 2011, p.54).

En su programa de gobierno, la Presidenta Bachelet también adhirió al paradigma de las industrias culturales, proponiendo aumentar "el financiamiento de los programas de fomento del arte y la cultura, para dar un impulso a las industrias culturales y a lo que hoy se llama 'economía creativa'". Entre otras medidas, propuso crear el Plan Nacional de Fomento de la Industria y Economía Creativa chilena y un comité multisectorial para coordinar las acciones de fomento provenientes de distintos organismos del Estado (Chile de Todos. Programa de Gobierno Michelle Bachelet 2014-2018, 2013, p.134).

Al igual que el giro desde la competitividad al desarrollo, es posible visualizar un cambio de énfasis en el paradigma de las industrias creativas. En la edición número 25 de Observatorio Cultural ${ }^{12}$ se alude al "debate que distingue el cambio de un paradigma de desarrollo cultural hacia uno de cultura para el desarrollo (Rodríguez, 2014, p.9) y se incluyen dos artículos que cuestionan la noción de industria cultural.

En el primero, Tomás Ariztía sostiene que conceptos como industrias creativas vuelven problemáticos los límites del campo cultural y los objetivos de las políticas culturales. Asimismo advierte sobre los riesgos de aplicar a la cultura el lenguaje y los métodos propios de la economía y del mercado -mecanismos y dispositivos de evaluación, prácticas propias del managment empresarial y la concepción de las audiencias como consumidores(Ariztía, 2014, p.114-119). Por su parte, Jaron Rowan critica que las políticas culturales inspiradas en este paradigma no promuevan la cultura por su valor intrínseco, sino que la instrumentalicen en función de otros objetivos: generación de empleo, promoción del consumo, etcétera. En esta lógica las formas de apoyo estatal pasan de la subvención a la promoción del emprendimiento. El autor expone diversos datos que darían cuenta del fracaso del modelo, el que a la larga habría generado inequidad, vulnerabilidad y precariedad laboral. Finalmente da cuenta de diversos movimientos ciudadanos que, en distintas ciudades europeas, comenzaron a manifestarse en contra de diversas iniciativas relacionadas con el modelo de las industrias creativas (Rowan, 2014, pp.120-127).

Resulta evidente que la amplitud de la definición de "cultura" trae dificultades al momento de operacionalizar el término. La actual Administración confirma la Declaración de México (1982), que considera la cultura, en su sentido más amplio, como

El conjunto de los rasgos distintivos, espirituales y materiales, intelectuales y afectivos que caracterizan a una sociedad o un grupo social. Ella engloba, además de las artes y las letras, los modos de vida, los derechos fundamentales al ser humano, los sistemas de valores, las tradiciones y las creencias. (CNCA, 2014a, p.8)

Así, la forma en que una sociedad concibe la salud es de naturaleza cultural, de la misma manera que la forma de entender las relaciones económicas o el rol que se le asigna a los adultos mayores. Sin embargo, resultaría absurdo delegar la gestión de la salud, de la economía y los programas de apoyo para adultos mayores, a un Ministerio de Cultura. 
Mientras más amplio sea el concepto de cultura, mayor es la cantidad de sectores incluidos en ella y mayor la diversidad entre sectores. Ello requiere políticas diferenciadas de acuerdo a la naturaleza y nivel de desarrollo de cada disciplina o sector. ${ }^{13}$ Si bien el diseño puede compartir categorías teóricas, métodos y hasta tiene una vinculación histórica con las artes visuales, la artesanía y la arquitectura, no es ni arte, ni artesanía, ni arquitectura. En gran medida, la discusión acerca de los efectos nocivos que una visión economicista puede tener en la cultura, es razonable -y hasta necesariaen el caso de los sectores artísticos nucleares -como las artes visuales, artes escénicas, música y literatura-, pero no es aplicable de la misma manera al ámbito del diseño, porque desde su origen como disciplina ha estado vinculado al sector productivo, constituyéndose en un mediador "entre necesidades y objetos, entre producción y consumo" (Maldonado, 1993, p.14). Dicho de otra manera: es razonable discutir la concepción de la cultura como recurso económico -y del producto cultural como bien de consumo- pero no lo es en el caso del diseño: el diseño es una aproximación a la solución de problemas y puede aplicarse tanto en el sector público como privado, para impulsar la innovación de productos, servicios e incluso en el diseño de políticas públicas; poniendo a las personas en primer lugar (Whicher, Swiatek, \& Cawood, 2015, p.4). Está referido a una realidad externa a sí mismo. El "para qué" del diseño no es la expresión artística -aunque esta pueda contribuir a mejorar la experiencia de un usuario, a comunicar significados o a generar identidad-.

Existe una amplia gama de ámbitos de desempeño en el diseño y una gama igualmente amplia de recursos metodológicos, técnicos y formales -desde aquellos que se centran en la expresión estética hasta los que atienden a factores de interacción y usabilidad; desde aquellos que vehiculan contenidos simbólicos hasta los que se orientan a reducir la complejidad cognitiva de información compleja y datos abstractos-; sin embargo, el diseño siempre es un recurso referido a una realidad externa a sí mismo: su objetivo no es el diseño. Concebido para el puro disfrute estético (mirar un lindo libro, disfrutar de una bella lámpara) es válido y también puede llegar a ser un buen negocio, pero esas funcionalidades no agotan las posibilidades del diseño y difícilmente podrán tener la capacidad de impactar significativamente a una sociedad en vías de desarrollo.

Al igual que las obras de arte, los productos diseñados pueden promover la reflexión crítica - no hay más que mirar el exprimidor de Starck o la tetera imposible de Jacques Carelman-, pero la reflexión no es el objetivo del diseño (aunque la reflexión sea una competencia muy necesaria en las fases analíticas del proyecto y para formar a un diseñador con capacidad crítica y con sentido de la responsabilidad cívica o social).

Las particularidades del diseño han sido claramente reconocidas por el CNCA, a través del Mapeo de las Industrias Creativas en Chile (CNCA, 2014b), publicado en enero de $2014^{14}$. El documento analiza en detalle los doce sectores que forman parte del CNCA, ${ }^{15}$ considerando aspectos relativos a la formación, creación y producción, comercialización y difusión, consumo y programas de financiamiento público. En relación al diseño, el estudio sostiene que "es uno de los sectores que más nítidamente permite visibilizar su funcionamiento como industria". Reconoce su carácter mediador en la medida que lo considera un instrumento al servicio de las necesidades de una sociedad o un cliente, usuario o consumidor. Lo caracteriza como el sector "que mayor enca-
13. Hasta la fecha se han formalizado políticas sectoriales para 8 de los 12 sectores creativos que forman parte del CNCA: Cine y Audiovisual, Libro y la Lectura, Música, Danza, Fotografía, Artes Visuales, Artesanía y Teatro.

14. El estudio fue publicado dos meses antes del cambio de gobierno, siendo Roberto Ampuero ministro presidente del CNCA.

15. Artesanía, Artes Visuales, Fotografía, Teatro, Danza, Artes Circenses, Editorial, Música, Audiovisual, Arquitectura, Diseño y Nuevos Medios. 
16. El 2 de agosto de 2016 la Cámara de Diputados aprobó en primer trámite constitucional el Proyecto de Ley que crea este esperado ministerio.

17. La Mesa técnica estuvo compuesta por Katherine Mollenhauer y Janio Thomas (miembros del Consejo Asesor en Diseño), Rodrigo Gajardo (diseñador experto en métricas), Gonzalo Brahm (representante del área de innovación del Ministerio de Economía), Rodrigo Ramírez (en ese momento, director del Magíster en Diseño Avanzado, Pontificia Universidad Católica de Chile) y Pablo Gálvez (asesor metodológico). denamiento presenta con otros sectores creativos y con otros sectores de la economía en general" y visualiza su potencial de crecimiento al considerarlo "un insumo y complemento de la producción de la mayor parte de los sectores económicos nacionales" (CNCA, 2014b, pp.225-237).

Esta condición fronteriza del diseño requiere de políticas de fomento diferenciadas, que no conlleven un castigo a su vinculación con la industria y los sectores productivos, pero que a su vez promuevan su aporte en el ámbito de la salud, de la educación, transportes, discapacidad, además de la cultura. Por la misma razón, resulta fundamental que en el organigrama del esperado Ministerio de la Cultura, las Artes y el Patrimonio, Diseño permanezca como una área independiente. ${ }^{16}$

Frente a la pregunta ¿diseñar bienes de consumo o bienes públicos?, la respuesta es: jambos!

\section{Política Nacional de Diseño}

Las políticas nacionales de Diseño han significado un componente clave en las estrategias que los países más desarrollados han adoptado para alcanzar mayores niveles de actividad económica y desarrollo social (Mollenhauer, 2014).

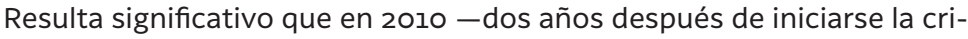
sis económica en Europa-, el diseño haya sido incluido en la Política de Innovación Europea, lo que ha permeado a nivel de sus países miembro. Actualmente 15 de los 28 países que conforman la Comunidad Europea han incorporado al diseño de forma explícita en sus políticas de innovación. La European Commission's Action Plan for Design-driven sostiene que un uso más sistemático de diseño como una herramienta para la innovación centrada en los usuarios y orientada al mercado en todos los sectores de la economía, complementarios a la I+D, pemitiría mejorar la competitividad de la Comunidad Europea (Whicher, Swiatek \& Cawood, 2015, p.4).

Desde 2013 el Área de Diseño del CNCA está trabajando en la elaboración de una Política Nacional de Diseño que, tras sucesivas postergaciones y retrasos, sería presentada a mediados de 2017. Según Figueroa, el retraso se ha debido tanto a la reestructuración de los sectores artísticos del CNCA, como a nuevas exigencias metodológicas del Departamento de Estudios del Consejo. En 2015 la propuesta de la entonces ministra Barattini proponía reunir en un solo sector a Diseño, Artes Visuales, Nuevos Medios y Fotografía, lo cual generó una fuerte oposición en el medio profesional y académico del diseño nacional. Una vez que se restablece su independencia como sector, el área de Diseño retoma el trabajo de la política, pero debe atender a nuevas exigencias orientadas a realizar consultas ciudadanas y regionales que permitan validar la propuesta. De materializarse esta iniciativa, verá la luz diez años después de un primer intento fallido -aunque no por ello menos meritorio- que llevó a cabo en 2007 un grupo de diseñadores y académicos. Aquella primera propuesta de política fue presentada al entonces ministro de Economía, Alejandro Ferreiro, durante el evento Mipyme Diseña: Innovar, Crecer, Competir, sin que prosperara su implementación (Mollenhauer, 2013).

El trabajo liderado por el CNCA durante 2013 se organizó sobre la base de cinco ejes estratégicos: Innovación y emprendimiento, Identidad y patrimonio, Internacionalización, Técnica y tecnología y Desarrollo territorial. Incluyó una mesa de expertos, ${ }^{17} \mathrm{y}$ una serie de sesiones organizadas bajo el modelo "consulta a expertos" en las que participaron diversos representantes del diseño nacional, tales como: escuelas de diseño, diseñadores de reconocida 
trayectoria, empresas y asociaciones gremiales, historiadores, medios de comunicación especializados, centros de innovación, entre otros.

En ambos procesos se incorporaron criterios estratégicos del modelo Sistema de Innovación Design_driven (SID_d), que permite mapear los habilitantes de una política de innovación guiada por el diseño. Es a su vez un sistema clasificatorio que indica niveles de madurez del Sistema Diseño de un país y permite visualizar un estado ideal al que deben estar orientadas estrategias de fomento del diseño (Mollenhauer, 2014).

Según Manuel Figueroa "la política está prevista y visualizada no solo como una política que intenta colocar un marco de principios, objetivos y tareas para el desarrollo de la disciplina, sino que también como un catalizador del desarrollo nacional en los más diversos ámbitos, permitiendo que el diseño pueda ser un generador de bienes públicos". ${ }^{18}$

Según Katherine Mollenhauer - que participó en ambos procesos como experta metodológica-, el trabajo liderado por el CNCA se caracterizó por un importante nivel de participación, ya que gracias a la organización en mesas de discusión se logró una convocatoria superior al centenar de personas, que representaban distintos sectores del diseño.

Esto es confirmado por Rodrigo Ramírez, quien destaca la participación de escuelas de Diseño de todo el país, lo que permitió recoger valiosos testimonios respecto de la realidad de la disciplina en regiones. En relación con la presencia de representantes de sectores productivos donde el diseño podría aportar significativamente, el académico sostiene que aún persiste una mirada reticente frente al diseño, lo que genera dificultades para lograr una mejor convocatoria.

\section{Conclusiones}

Hace cincuenta años, Ulm inició un camino para consolidar la actividad proyectual como dominio disciplinar, lo que implicó alejarse del paradigma de las artes y acercarse hacia ámbitos del saber vinculado a las ciencias humanas, las ciencias exactas y la tecnología. Ello iba de la mano de un plan de estudios que debía responder a las necesidades de reconstrucción material y moral, así como a estrategias de vinculación con distintos sectores del ámbito académico y productivo. Este modelo permite orientar el diseño de programas académicos, pero también podría contribuir al diseño de políticas de fomento del diseño, ya que responde a preguntas fundamentales como: ¿cuál es el tipo de sociedad en la que queremos vivir?, ¿de qué manera un diseñador puede contribuir a ello?, ¿cuál es el perfil del diseñador que deberá responder a las necesidades de dicha sociedad?, ¿cuáles son los actores relevantes de la disciplina y cómo generar sinergias en beneficio de esta? En los últimos años se ha avanzado en iniciativas centradas en la difusión y promoción del diseño chileno. Con ello se ha alcanzado importantes logros como la realización de bienales y exposiciones, la participación de diseñadores chilenos en renombrados eventos internacionales de diseño, la incorporación de una línea de concurso exclusiva en Fondart, por nombrar solo algunas. Esto ha sido el resultado de un esfuerzo sistemático por parte de universidades, institutos y asociaciones de profesionales y de empresas de diseño. El Área de Diseño del CNCA ha cumplido un rol fundamental en este sentido, coordinando y apoyando iniciativas, convocando y generando oportunidades de participación.

Las medidas orientadas a fomentar la creación, la difusión y el emprendimiento creativo, han sido favorables y deben sostenerse, pero ha llegado el
18. Entrevista realizada en marzo de 2015, vía correo electrónico. 
momento de que los esfuerzos también apunten a promover la integración del diseño al tejido social, a generar impacto a través del diseño. El foco no puede seguir siendo solo el desarrollo del diseño como sector creativo. El giro desde la competitividad hacia el desarrollo debería abrir nuevas oportunidades para el diseño chileno, siempre que implique avanzar hacia nuevas problemáticas, sin desatender los territorios conquistados hasta ahora. Iniciativas como el Plan Nacional para la Economía Creativa, el Laboratorio de Gobierno y el diseño de una Política Nacional de Diseño, constituyen importantes señales en relación al valor que el Estado chileno le estaría dando al diseño. Podrán llegar a ser una verdadera oportunidad para el desarrollo de la disciplina en el país, en la medida que el primero no demonice su rol en las dinámicas de mercado y que el segundo lo incorpore en fases tempranas de los procesos de evaluación y diseño de servicios públicos. Seremos testigos de una nueva etapa en el diseño chileno, en la medida en que se incrementen iniciativas articuladas que promuevan su incorporación sistemática y progresiva a un mayor número de sectores como Educación, Salud, Desarrollo Social, Minería y Agricultura. Es necesario reconocer que el diseño no solo agrega valor mediante estrategias de diferenciación, sino que también contribuye a hacer más eficiente diversos procesos y servicios. Las mesas interministeriales pueden tener un rol clave en este sentido.

Esta nueva etapa también impone nuevos desafíos para la profesión y para las universidades e institutos, que deberán ponerse al día con una formación que desarrolle competencias de estrategia, interdisciplina, gestión, metodología e investigación.

Si hemos logrado que economistas reconozcan que es necesario incorporar al diseño en etapas tempranas de las cadena de valor, si hemos conseguido que el Estado incorpore al diseño en su programa de modernización, entonces los futuros diseñadores deberán ser los primeros en estar convencidos que pueden hacerlo y que tienen las competencias necesarias. La lógica del encargo que se da en las escuelas de diseño - donde un proyecto comienza con un brief perfectamente delineado y culmina con la presentación de una propuesta formal-, ya no responde a la formación en diseño. Los estudiantes tienen que lograr, en primer lugar, identificar problemas $-\mathrm{y}$ no cualquier problema, sino aquellos que son pertinentes a la disciplina-y luego proponer un plan de acción en el que se defina con claridad cuáles son los objetivos y efectos esperados, cuáles los recursos involucrados y cuáles los indicadores de evaluación de impacto. La formulación y evaluación de proyectos es un imperativo de la enseñanza del diseño. Las universidades tenemos las misión de contribuir al desarrollo disciplinar fomentando la investigación en el campo histórico, metodológico y teórico. Mejorar la formación teórica, mejorar la reflexión, la metodología y las competencias para la investigación. Solo de esta manera el diseño podrá contribuir a mejorar la calidad de vida de las personas y hacer de Chile un país más habitable y de esta, una sociedad más amable. 


\section{Bibliografía}

Aicher, O. (1997). El mundo como proyecto. Barcelona: Gustavo Gili.

Ariztía, T. (2014). Cultura y economía: itinerario de dos conceptos. Observatorio Cultural, (25), 114-119.

Bonsiepe, G. (1999). Del objeto a la interfase. Buenos Aires: Ediciones Infinito.

Bonsiepe, G. (2003). El discurso de Ulm. En D. Rinker, M. Quijano y B. Reinhardt, Modelos de Ulm - modelos post-UIm | hochschule für gestaltung UIm 1953-1968 (pp. 15-17). Stuttgart: HfG-Archiv/Ulmer Museum.Bonsiepe, Gui (2003). Sobre la relevancia de la HfG Ulm. En D. Rinker, M. Quijano y B. Reinhardt, Modelos de Ulm - modelos post-UIm | hochschule für gestaltung UIm 1953-1968 (pp. 22-25). Stuttgart: HfG-Archiv/Ulmer Museum.

Chile de Todos. Programa de Gobierno Michelle Bachelet 20142018. (2013). Santiago de Chile. Recuperado de http:// michellebachelet.cl/programa/ (Marzo de 2015).

Comisión Nacional de Innovación para la Competitividad, CNIC (2013). Orientaciones estratégicas para la innovación. Surfeando hacia el futuro. Chile en el horizonte 2025. CNIC. Recuperado de http://www.innovacion. gob.cl/wp-content/uploads/2013/o8/orientaciones_ estrategicas.pdf (Diciembre de 2014).

Comisión Propuesta de la Política Nacional de Diseño (2007). Propuesta de Política Nacional de Diseño. Resumen Ejecutivo. Consejo Nacional de la Cultura y las Artes, CNCA (2005). Chile quiere más Cultura. Definiciones de Política Cultural 2005-2010. Santiago de Chile: CNCA. Recuperado de http://www.cultura.gob.cl/wp-content/ uploads/2012/03/Chile-Quiere-M\%C3\%Als-Cultura.-Definiciones-de-Pol\%C3\%ADtica-Cultural-2005-2010.pdf (Marzo de 2015).

Consejo Nacional de la Cultura y las Artes, CNCA (2011). Política Cultural 2011-2016. Valparaíso: CNCA. Recuperado de http://www.cultura.gob.cl/wp-content/uploads/2011/11/ politica_cultural_2011_2016.pdf (Marzo de 2015).

Consejo Nacional de la Cultura y las Artes, CNCA (2014). Contenidos de la Indicación Sustitutiva del Proyecto de Ley que crea el Ministerio de Cultura. Presentación, Santiago de Chile.

Consejo Nacional de la Cultura y las Artes, CNCA (2014). Mapeo de las industrias creativas en Chile. Caracterización y dimensionamiento. Santiago de Chile: CNCA. Recuperado de http://www.cultura.gob.cl/wp-content/uploads/2014/o1/mapeo_industrias_creativas. pdf (Diciembre de 2014).

Fernández, S. (2003). La influencia de la Hfo Ulm en la enseñanza de diseño en América Latina. En D. Rinker, M. Quijano y B. Reinhardt, Modelos de Ulm - modelos post-UIm | hochschule für gestaltung UIm 1953-1968 (pp. 18-21). Stuttgart: HfG-Archiv/Ulmer Museum.
Lindinger, H. (1991). Ulm Design. The Morality of Objects. Cambridge, Mass.: MIT Press.

Maldonado, T. (1977). Vanguardia y racionalidad. Artículos, ensayos y otros escritos 1946-1974. Barcelona: Gustavo Gili.

Maldonado, T. (1993). El diseño industrial reconsiderado (за ed.). Barcelona: Gustavo Gili.

Mäntele, M. (2003). Magos de la teoría. En D. Rinker, M. Quijano y B. Reinhardt, Modelos de Ulm - modelos post-UIm | hochschule für gestaltung UIm 1953-1968 (pp. 13-14). Stuttgart: HfG-Archiv/Ulmer Museum.

Mollenhauer, K. (2013). El Sistema de Innovación Design_ driven como modelo estratégico y la propuesta de Política Nacional de Diseño para Chile 2007. Revista Chilena de Diseño, (3), 23-47.

Mollenhauer, K. (2014). Modelos y metodologías design_ driven para el desarrollo estratégico de la innovación y el emprendimiento social. Ponencia.

Poblete, J. (2015). Creatividad en Chile: ¿los peores del barrio? Capital, (389), 20-24.

Quijano, M. (2003). ¿Será el camino la meta? En D. Rinker, M. Quijano y B. Reinhardt, Modelos de UIm - modelos post-UIm | hochschule für gestaltung Ulm 1953-1968 (pp. 10-12). Stuttgart: HfG-Archiv/UImer Museum.

Piketty, T. (2014). El capital en el siglo XXI, La Gaceta del Fondo de Cultura Económica, (523), 6-10.

Programa de las Naciones Unidas para el Desarrollo, PNUD y Naciones Unidas para la Educación, la Ciencia y la Cultura, UNESCO (2013). Informe sobre la Economía Creativa. Edición especial 2013. Ampliar los cauces del desarrollo local. México: Naciones Unidas/PNUd/UNESCO. Recuperado desde http://www.unesco.org/culture/pdf/creative-economy-report-2013-es.pdf (Enero de 2015).

Rinker, D. (2003). “El diseño de productos no es arte” - El aporte de Tomás Maldonado al surgimiento de un nuevo perfil profesional. En D. Rinker, M. Quijano y B. Reinhardt, Modelos de Ulm - modelos post-Ulm | hochschule für gestaltung UIm 1953-1968 (pp. 5-9). Stuttgart: HfG-Archiv/Ulmer Museum.

Rodríguez, R.M. (2014). Editorial. Observatorio Cultural, (25), 9. Rowan, J. (2014). La cultura como problema: ni Arnold ni Florida. Reflexiones acerca del devenir de las políticas culturales tras la crisis. Observatorio Cultural, (25), 120-127. Sapag, R. (2015). Innovar o morir. Capital, (389), 12. Whicher, A., Swiatek, P. \& Cawood, G. (2015). Design Policy Monitor 2015. Reviewing Innovation and Design Policies Across Europe. Cardiff: SEE Platform. Recuperado desde http://www.seeplatform.eu/docs/SEE\%20DPM\%20 2015\%20Jan.pdf (Marzo de 2015). 